\title{
VARIABILIDADE ESPACIAL E PREENCHIMENTO DE FALHAS DE DADOS PLUVIOMÉTRICOS PARA O ESTADO DE ALAGOAS
}

\author{
HENDERSON SILVA WANDERLEY ${ }^{1}$, RICARDO FERREIRA CARLOS DE AMORIM ${ }^{2}$, FREDE \\ OLIVEIRA DE CARVALHO ${ }^{3}$
}

\author{
${ }^{1}$ Universidade Federal de Viçosa (UFV), Departamento de Engenharia Agrícola, Viçosa, MG, Brasil \\ ${ }^{2}$ Universidade Federal de Alagoas (UFAL), Instituto de Ciências Atmosféricas, Maceió, AL, Brasil \\ ${ }^{3}$ UFAL, Unidade Acadêmica de Tecnologia, Maceió, AL, Brasil
}

henderson.wanderley@ufv.br; rfcamorim@gmail.com; fredecarvalho@yahoo.com.br

Recebido Agosto de 2011 - Aceito Dezembro 2011

\begin{abstract}
RESUMO
A região tropical é marcada por apresentar grande variabilidade quanto à distribuição dos seus regimes pluviométricos, e o conhecimento dessa variabilidade torna-se fundamental para obtenção dos padrões definidores dos regimes hidrológicos e climatológicos desta região. No entanto, a falta de informação quanto à distribuição da precipitação é um sério obstáculo para se compreender e modelar sua variabilidade, surgindo a necessidade de se obter informações para regiões que não apresentam estações de medições ou que apresentem falhas em seu banco de dados através da interpolação. O método consiste em utilizar técnicas geoestatísticas na compreensão da variabilidade espacial e no preenchimento de falhas de dados pluviométricos para o Estado de Alagoas. Para o estudo foram selecionados dados de 63 estações pluviométricas, provenientes da Agência Nacional de Águas (ANA) entre os anos de 1965 a 1980. A utilização da geoestatística, com a análise variográfica, mostrou que as características estruturais estudadas da precipitação estão correlacionadas e apresentam forte dependência espacial. A estimativa da precipitação obtida pelo método de Krigagem Ordinária apresentou resultados satisfatórios, para a distribuição espacial da precipitação, bem como para o preenchimento de falhas.
\end{abstract}

Palavras chaves: Geoestatística, krigagem ordinária, preenchimento de falhas.

\begin{abstract}
SPATIAL VARIABILITY AND MISSING PRECIPITATION DATA FILLING IN ALAGOAS STATE

Tropical region is characterized by great variability in the distribution of the rainfall regimes, and knowledge of this variability is fundamental to achieve the standards that define the climatologic and hydrological regimes of this region. However, lack of information regarding the distribution of rainfall is a serious obstacle to understanding and modeling its variability, resulting in the need to obtain information for regions that do not have measuring stations or shows flaws in its database, by interpolation. The method uses geostatistic technique to understand the spatial variability and on the filling of rainfall data missing on the Alagoas state. For the study 63 rainfall gauge stations of the Agência Nacional de Águas (ANA) in the period 1965 to 1980 were selected. The use of geostatistic with variographic analysis showed that the structural characteristic of the studied rainfall are correlated and have strong spatial dependence. The estimation of rainfall obtained by ordinary kriging method showed satisfactory results for the spatial distribution of rainfall as well as in filling the missing data values.
\end{abstract}

Keyword: Geostatistic, ordinary kriging, missing precipitation. 


\section{INTRODUÇÃO}

A região tropical é marcada por apresentar grande variabilidade quanto à distribuição dos seus regimes pluviométricos. Nestas localidades, a precipitação determina as chamadas estações secas e chuvosas, e o conhecimento de sua variabilidade espacial e temporal torna-se fundamental para obtenção dos padrões definidores dos regimes hidrológicos e climatológicos desta região.

A representação dessa distribuição, tanto espacial quanto temporal, é essencial para determinar a influência da precipitação no ciclo hidrológico, na agricultura, assim como no turismo, dentre outras atividades. No entanto, a falta de informação torna-se um obstáculo para determinar sua espacialização, e consequentemente, modelar sua variabilidade. Essas lacunas são causadoras de grande incerteza nos processos de estimativa e compreensão da distribuição dessa variável. Sirois (1990) mostrou que incertezas na interpolação de dados de precipitação é um sério obstáculo para se compreender os processos hidrológicos e meteorológicos, devido à falta de dados. $\mathrm{O}$ autor ressalta que a utilização de dados que apresentaram falhas pode ser tendenciosa, se o valor da amostra com falha for muito diferente do possível valor que seria observado, onde o erro absoluto e o desvio-padrão tornam-se maior à medida que aumenta a diferença entre as amostras.

Teegavarapu e Chandramouli (2005) enfatizaram que nos tratamentos de dados meteorológicos é frequente o fato de várias estações apresentarem falhas em seu banco de dados. Deste modo, a interpolação espacial e/ou temporal surge como uma etapa primordial no estudo da distribuição da precipitação, principalmente, quando se trata de regiões que não apresentam estações de medições ou que tenham falhas em seu banco de dados. Uma vez que, o método de interpolação permite construir informações a partir de um conjunto discreto de dados pontuais previamente conhecidos.

No entanto, o processo de interpolação de dados de precipitação permanece sendo uma tarefa complexa, principalmente para pequenas bacias hidrográficas, onde essas falhas dificultam a elaboração de estudos que busquem modelar sua variabilidade (Coulibaly e Evora, 2007). Os processos de estimativa de dados pluviométricos, por vários anos, foram obtidos por técnicas de regressão e modelos de séries temporais. Atualmente, diversas abordagens matemáticas, estatísticas e geoestatísticas, estão sendo usadas para a interpolação de dados de precipitação (Bargaoui e Chebbi, 2009).

A utilização da geoestatística como método de interpolação, para regiões que apresentam falhas, vem mostrando resultados satisfatórios no que concerne a variáveis regionalizadas como a precipitação (Haberlandt, 2007). Isto se deve ao fato da geoestatística considerar que a distribuição espacial da precipitação é definida por uma função aleatória, dentro da região de estudo. Com a utilização da geoestatística, após verificada a existência da dependência espacial através do variograma, estima-se a variável de interesse pelo o método interpolativo de krigagem.

Apesar da existência de outros métodos para gerar informações espaciais de medidas pontuais, a utilização da técnica de krigagem para o processo de interpolação vem mostrando resultados mais realistas do que outros métodos interpolativos comumente utilizados (Haberlandt, 2007). Segundo Bargaoui e Chebbi (2009) a krigagem se destaca dos outros métodos por possibilitar a análise da variância dos dados através da análise variográfica, a qual expressa a descontinuidade espacial dos dados.

Desta forma, o objetivo deste estudo consiste em utilizar a geoestatísticas na compreensão da variabilidade espacial e na interpolação de falhas de dados pluviométricos para o Estado de Alagoas.

\section{MATERIAIS E MÉTODOS}

O estudo foi realizado no Estado de Alagoas, o qual localiza-se na região leste do nordeste brasileiro, limitando-se ao norte com o estado de Pernambuco, ao sul com Sergipe, a oeste com Pernambuco e Bahia e a leste com o Oceano Atlântico. O Estado está dividido em seis microrregiões ambientais: Litoral, Zona da Mata, Agreste, Sertão, Sertão do São Francisco e Baixo São Francisco (Figura 1).

Foram selecionados dados mensais de 63 estações pluviométricas distribuídas espacialmente no estado de Alagoas (Figura 2), provenientes do banco de dados da Agência Nacional de Águas (ANA), compreendidos entre os anos de 1965 a 1980. O estudo foi realizado para os meses de maio e novembro, meses de máxima e mínima distribuição da precipitação no Estado. Para esses meses busca-se compreender a distribuição espacial da precipitação, bem como, estimar valores desta variável para as regiões que não apresentam estações de medição. Como esses meses apresentam os extremos pluviométricos, a obtenção de resultado satisfatório para interpolação, suprime a necessidade da realização para os demais meses, uma vez que a precipitação que será estimada está compreendida entre a mínima e a máxima observada nestes meses.

Duas abordagens foram utilizadas para a interpolação, uma com o uso de 63 estações e outra com 58, onde cinco estações foram retiradas do conjunto de dados, admitindo assim a existência de possíveis falhas. A exatidão dos valores estimados, pela técnica de krigagem, das estações que foram retiradas do conjunto de dados foi avaliada pelo Desvio Médio (DM) e pelo Erro Quadrático Médio (EQM) (Equações 1 e2, respectivamente). Esta abordagem foi proposta para avaliar se os valores estimados 


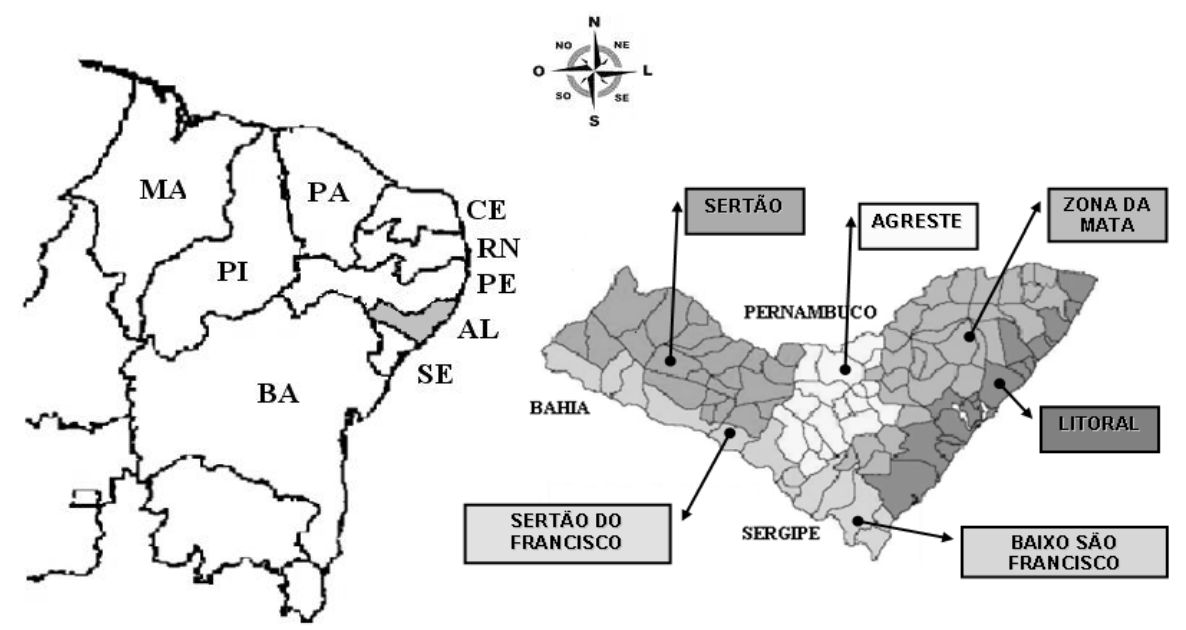

Figura 1 - Estado de Alagoas e suas microrregiões.

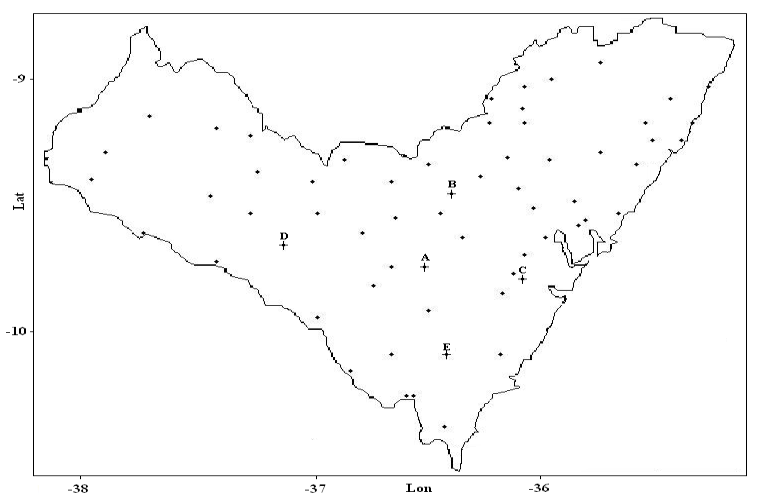

Figura 2 - Distribuição espacial das estações no Estado de Alagoas.

para as regiões que não dispõem de estações são coerentes, onde os valores interpolados, em muitos casos, são admitidos como reais para essas regiões, uma vez que uma análise qualitativa para esses dados é inexistente, decorrente da falta de informações nestas localidades. As estações retiradas do conjunto de dados foram classificadas em A, B, C, D e E (Figura 2), onde suas informações geográficas são apresentadas na Tabela 1 .

$$
\begin{aligned}
& D M=\sum_{i=1}^{n} \frac{\left|x_{i}-x_{e}\right|}{n} \\
& E Q M=\sqrt{\sum_{i=1}^{n} \frac{\left(x_{i}-x_{e}\right)^{2}}{n}}
\end{aligned}
$$

em que, $x_{i}$ valor observado na estação; $x_{e}$ valor estimado.

A geoestatística é definida como um conjunto de técnicas, que estima valores regionalizados e espacializados de variáveis ou elementos de uma determinada área em estudo. A geoestatística é uma metodologia que permite uma análise espacial e/ou temporal de dados correlacionados. A principal característica da geoestatística está na utilização do variograma (ou semi-variograma), o qual permite através de técnicas simples definir um modelo estrutural de correlação espacial de uma variável.

O variograma é a ferramenta básica para o procedimento de interpolação através do método de krigagem, pois o mesmo permite uma representação quantitativa da variação de um fenômeno regionalizado no espaço e no tempo (Carvalho et al. 2009). O variograma expressa a dependência espacial através da medida da variância dos valores amostrais, separados por uma distancia h, definido pela Equação 3.

$$
y(h) \frac{1}{2 N(h)}=\sum_{i=1}^{N(h)}[\mathrm{Z}(\mathrm{x} i)-\mathrm{Z}(\mathrm{x} i+\mathrm{h})]^{2}
$$

em que, $\mathrm{N}(\mathrm{h})=$ número de pares de valores amostrados $[\mathrm{Z}(\mathrm{xi})$ $-\mathrm{Z}(\mathrm{xi}+\mathrm{h})]$ separados pelo vetor $\mathrm{h}$.

Neste estudo foram utilizados os modelos isotrópicos experimentais de variograma do tipo esférico e exponencial, ambos com patamar, os quais vêm demonstrando resultados satisfatórios em estudos que utilizam precipitação (Zamboti, 2001). Um exemplo ilustrativo do variograma e seus parâmetros: patamar, efeito pepita, alcance e variância estrutural, é mostrado na Figura 3.

Tabela 1 - Informações geográficas das estações utilizadas para a interpolação espacial.

\begin{tabular}{ccccc}
\hline Estações & $\begin{array}{c}\text { Nome das } \\
\text { Estações }\end{array}$ & $\begin{array}{c}\text { Latitude } \\
\left({ }^{\circ}\right)\end{array}$ & $\begin{array}{c}\text { Longitude } \\
\left({ }^{\circ}\right)\end{array}$ & $\begin{array}{c}\text { Altitude } \\
(\mathrm{m})\end{array}$ \\
\hline $\mathrm{A}$ & $\begin{array}{c}\text { Limoeiro de } \\
\text { Anadia }\end{array}$ & -9.75 & -36.5 & 150 \\
\hline $\mathrm{B}$ & Mar Vermelho & -9.45 & -36.38 & 620 \\
\hline $\mathrm{C}$ & $\begin{array}{c}\text { Sebastião } \\
\text { Ferreira }\end{array}$ & -9.8 & -36.06 & 0 \\
\hline $\mathrm{D}$ & Batalha & -9.66 & -37.13 & 120 \\
\hline $\mathrm{E}$ & $\begin{array}{c}\text { Colônia } \\
\text { Pindorama }\end{array}$ & -10.11 & -36.4 & 110 \\
& & &
\end{tabular}




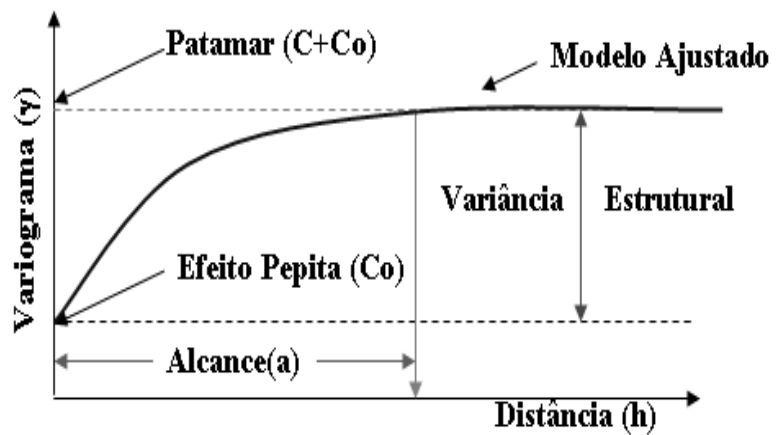

Figura 3 - Modelo de Variograma Experimental com Patamar.

O patamar $\left(\mathrm{C}+\mathrm{C}_{\mathrm{o}}\right)$ se refere ao ponto o qual a função se estabiliza no variograma, valor do variograma correspondente ao seu alcance (a). Deste ponto em diante, considera-se que não existe mais dependência espacial entre as amostras, porque a variância da diferença entre pares de amostras torna-se invariante com a distância.

O Alcance (a) é a distância dentro da qual as amostras apresentam-se correlacionadas espacialmente. Já o Efeito Pepita $\left(\mathrm{C}_{\mathrm{o}}\right)$ é o ponto onde o variograma toca o eixo das ordenadas. Esse ponto revela a descontinuidade do variograma para distâncias menores do que a menor distância entre as amostras. A Variância Estrutural (C) se refere a diferença entre o patamar $\left(C+\mathrm{C}_{\mathrm{o}}\right)$ e o efeito pepita $\left(\mathrm{C}_{\mathrm{o}}\right)$.

Verificada a dependência espacial, é possível estimar valores da precipitação para os locais não amostrados dentro do campo de análise através da técnica de Krigagem. Essa técnica usa informações a partir do variograma para encontrar os pesos a serem associados às amostras com valores conhecidos, que irão estimar pontos desconhecidos. O termo krigagem é derivado do nome Daniel G. Krige, que foi o pioneiro a introduzir o uso de médias móveis para evitar a superestimação sistemática de reservas de mineração. Inicialmente, o método de krigagem foi desenvolvido para solucionar problemas de mapeamentos geológicos, mas seu uso expandiu-se com sucesso para outras áreas como a meteorologia.

A krigagem pode ser entendida como uma série de técnicas de análise de regressão que procura minimizar a variância estimada a partir de um modelo prévio, que leva em consideração a dependência estocástica entre os dados distribuídos no espaço. Os principais tipos de krigagem são a simples, a ordinária e a universal. A krigagem ordinária (KO) foi escolhida para a interpolação neste estudo por apresentar resultados satisfatórios quando comparadas com os outros tipos de krigagem (Alsamamra et al., 2009).

A estimativa por krigagem ordinária busca por um conjunto de ponderadores que minimize a variância do erro da estimativa (Landim, 2006). O método de krigagem ordinária pode ser escrito em notação matricial como mostra a Equação 4.

$$
\mathrm{K} . \lambda=\mathrm{k} \quad \rightarrow \quad \lambda=\mathrm{k} \cdot \mathrm{K}^{-1}
$$

em que, $\mathrm{K}$ e $\lambda$ são matrizes das covariâncias (ou variogramas) e k o vetor dos pesos.

A vantagem principal da krigagem ordinária é que os dados usados descrevem o grau de influência visando atribuir pesos às observações. Lluyd (2005) comentou que os pesos são atribuídos arbitrariamente pela Equação 5.

$$
\mathrm{Z}\left(\mathrm{x}_{\mathrm{i}}\right)=\mathrm{f}\left(\mathrm{x}_{\mathrm{i}}\right)+\mathrm{e}\left(\mathrm{x}_{\mathrm{i}}\right), \mathrm{i}=1,2, \ldots, \mathrm{n}
$$

em que, $\mathrm{f}\left(\mathrm{x}_{\mathrm{i}}\right)$ = função aleatória $\mathrm{F}$ num ponto $\mathrm{x}_{\mathrm{i}} ; \mathrm{e}\left(\mathrm{x}_{\mathrm{i}}\right)$ são realizações médias de zero e erros. E a correlação espacial entre os pontos de medida pode ser quantificada por meio da função de variância (Equação 6).

$$
y(x, h)^{1} / 2 \operatorname{var}[F(x)-F(x+h)
$$

em que, assume que $\mathrm{h}$ é a distância euclidiana entre dois pontos e que a tendência é constante, e $\mathrm{y}(\mathrm{x}, \mathrm{h})$ são independentes de $\mathrm{x}$. Uma função paramétrica é usada para modelar a variância para valores diferentes de $\mathrm{h}$.

\section{RESULTADOS E DISCUSSÕES}

A distribuição mensal da precipitação no estado de Alagoas é caracterizada por apresentar grande variabilidade espacial. Essa distribuição possui dois períodos distintos na espacialidade das chuvas, um de máxima e outro de mínima, com alguns meses de transição. O período chuvoso está compreendido entre os meses de abril a julho, enquanto o período seco entre os meses de outubro a janeiro. Os meses de transição do período seco para o período chuvoso estão compreendidos entre os meses de fevereiro e março, enquanto que agosto e setembro são os meses de transição do período chuvoso para o período seco (Figura 4).

Após o mês de março são observadas precipitações máximas acima dos $140 \mathrm{~mm}$ até o mês de julho. Parte desses índices pluviométricos se deve a influência da umidade proveniente do oceano. Após o mês de julho a precipitação apresenta uma redução significativa em seus totais, onde o mês de outubro apresentou um valor de aproximadamente $50 \mathrm{~mm}$, marcando o início do período seco no Estado.

Entre os modelos de variogramas testados, o modelo esférico obteve melhor ajuste para o mês de maio e o exponencial para o mês de novembro (Tabela 2). O modelo esférico descreve uma curva mais suave de sua função apresentando resultados satisfatório para os meses com índices pluviométricos elevados como no trabalho de Kuhn et al. (2007). O baixo valor encontrado para o efeito pepita, em relação ao patamar, demonstra que na série utilizada de dados não há erros significativos de medições. $\mathrm{O}$ alcance para esse mês determina uma região de dependência espacial de $72 \mathrm{Km}$. 


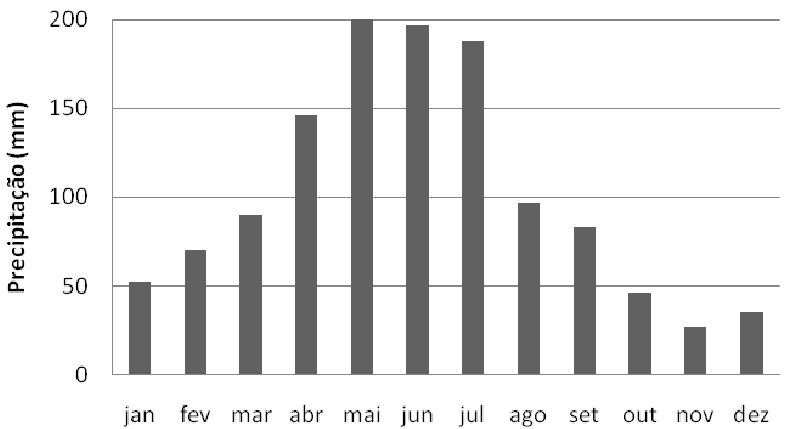

Figura 4 - Média mensal da precipitação no Estado de Alagoas de 1965 a 1980.

Tabela 2 - Parâmetros dos modelos teóricos ajustados ao variograma experimental para a precipitação média em Alagoas. $\left(\mathrm{C}_{0}=\right.$ Efeito Pepita, $\mathrm{C}+\mathrm{C}_{0}=$ Patamar, $\mathrm{a}=$ Alcance, em Km)

\begin{tabular}{ccccc}
\hline Meses & $\begin{array}{c}\text { Modelo de } \\
\text { variograma }\end{array}$ & $\mathrm{C}_{\mathrm{o}}$ & $\mathrm{C}+\mathrm{C}_{\mathrm{o}}$ & $\mathrm{a}$ \\
\hline Maio & Esférico & $1.2881 \mathrm{e}^{-10}$ & 1.2769 & 72 \\
\hline Novembro & Exponencial & 0.1105 & 2.4472 & 80 \\
\hline
\end{tabular}

Para o mês de novembro o modelo exponencial de variograma demonstrou melhor ajuste aos dados de precipitação. Esse modelo atinge o patamar assintoticamente, enquanto que o modelo esférico o atinge no valor do alcance. Para este tipo de variograma a literatura recomenda utilizar apenas $95 \%$ do valor do patamar (Shao et al., 2007). O efeito pepita obtido por este modelo, semelhante ao mês de maio, demonstrando resultado satisfatório e indicando a ausência de falhas que comprometessem a utilização dos dados.

A região de dependência espacial obtida por esse modelo demonstrou um alcance de $80 \mathrm{Km}$, uma vez que a literatura não recomenda utilizar $100 \%$ desse valor. A região de dependência espacial para esse mês foi cerca de $76 \mathrm{Km}$, resultado próximo do valor encontrado para o mês de maio. É bom ressaltar que, em vários estudos que envolvem eventos naturais, o modelo esférico e exponencial são os que melhor expressam os resultados, destacando-se entre esses a precipitação pluviométrica (Lee et al., 2006).

Mello et al. (2003) trabalhando com dados de precipitação, encontraram alcance que variaram de 90 a $100 \mathrm{Km}$, já o encontrado por Haberlandt (2007) oscilaram de 80 a $140 \mathrm{Km}$, mostrando coerência com os resultados obtidos neste estudo. Essa distância expressa a área máxima de influência da variável em estudo, onde após essa distância, as amostras tornam-se independentes, não sendo mais caracterizada a dependência espacial. Assim, os ajustes obtidos através dos variogramas testados neste estudo, apresentaram resultado satisfatório no ajuste de seus parâmetros. Cambardella et al. (1994) consideraram que um bom ajuste e uma forte dependência espacial são obtidos quando o efeito pepita é inferior a $25 \%$ do valor do patamar.

Haberlandt (2007) encontrou efeito pepita que variou de 10 a $40 \%$ do patamar. Resultado análogo ao encontrado por Teegavarapu (2007), que obteve o efeito pepita entre $10 \mathrm{e}$ $20 \%$ em relação ao patamar. Estes estudos validam a eficácia dos resultados encontrados neste estudo, onde os índices obtidos foram inferiores a $5 \%$ valor do patamar, demonstrando forte dependência espacial entre os dados de precipitação pluviométrica utilizados.

A distribuição da precipitação para o mês de maio demonstra que os maiores índices pluviométricos estão localizados nas regiões da grande Maceió e Litoral Norte, apresentando totais de 250 a $320 \mathrm{~mm}$, respectivamente (Figura 5). A precipitação pluviométrica mostrou uma significativa redução para as demais áreas do Estado, atingindo $200 \mathrm{~mm}$ para as localidades do Sertão e do Sertão do São Francisco. Para este mês, a região do Agreste foi caracterizada como o divisor de água, com precipitação variando de 150 a $250 \mathrm{~mm}$. Em seguida, observou-se que os totais pluviométricos foram inferiores a $100 \mathrm{~mm}$, caracterizando parte expressiva do Sertão e do Sertão do São Francisco.

De acordo com Molion e Bernardo (2002), o mês de maio está inserido na quadra chuvosa do leste do Nordeste, que compreende os meses de abril, maio, junho e julho, sendo esse o período mais úmido. Nos anos mais chuvosos, esses índices chegam a representar cerca de 60 a $70 \%$ de toda a precipitação que atinge a região, os quais são decorrentes do deslocamento gradativo da Zona de Convergência do Atlântico Sul (ZCAS), alimentada pela corrente de umidade trazida pelo vento Alísio, intensificando os sistemas de brisa e a formação de células convectivas que penetram mais sobre o continente. Os meses que estão inseridos na quadra chuvosa do estado de Alagoas mostram grande potencialidade para o cultivo agrícola.

Os resultados obtidos na estimação da precipitação para as estações A, B, C, D e E, para os meses em estudo, não mostraram diferença significativa entre os totais observados e os estimados pelo método de krigagem ordinária (Tabela 3). Deste modo, os valores de precipitação estimados para as

Tabela 3 - Precipitação (mm) observada (obs) nas estações de medições e estimada (Est) pelo método de Krigagem Ordinária para os meses de maio e novembro.

\begin{tabular}{ccccc}
\hline \multirow{2}{*}{ Estações } & \multicolumn{2}{c}{ Maio } & \multicolumn{2}{c}{ Novembro } \\
\cline { 2 - 5 } & Obs & Est & Obs & Est \\
\hline A & 168,6 & 167,3 & 18,7 & 18,1 \\
\hline B & 204,8 & 204,2 & 18,8 & 18,4 \\
\hline C & 104,0 & 108,4 & 3,9 & 5,2 \\
\hline D & 104,0 & 103,8 & 15,7 & 15,5 \\
\hline E & 264,5 & 258,9 & 35,7 & 31,3 \\
\hline
\end{tabular}


regiões que não apresentam estações de medições também foram satisfatórios.

Para as estações $\mathrm{C}$ e $\mathrm{E}$ foram evidenciados os maiores erros entre os valores observados e estimados. Este acontecimento é decorrente da grande variabilidade pluviométrica apresentada no mês de maio para as regiões próximas às estações $\mathrm{C}$ e $\mathrm{E}$. Entretanto, essa diferença não apresentou uma variação maior que $6 \mathrm{~mm}$ entre os valores observados e estimados (Figura 6). O desvio médio e o erro quadrático médio calculado para este mês foi de 2,4 mm e de 3,2 $\mathrm{mm}$, respectivamente.

A distribuição espacial da precipitação, para o mês de novembro em Alagoas, mostra que no Estado, em quase toda sua totalidade, os índices pluviométricos oscilaram entre $10 \mathrm{e}$ $40 \mathrm{~mm}$ (Figura 7). Esses totais incluem o Sertão, Sertão do São Francisco, Agreste, parte do Litoral e Zona da Mata do estado de Alagoas. Os maiores índices de precipitação, para este mês, foram observados no nordeste do estado de Alagoas, chegando a valores acima de $70 \mathrm{~mm}$. O mês de novembro está compreendido entre os meses mais secos, os quais se estendem entre os meses de outubro, novembro, dezembro e janeiro. Esses meses são marcados por apresentar baixos índices pluviométricos, o que pode ocasionar perdas agrárias expressivas para o Estado.

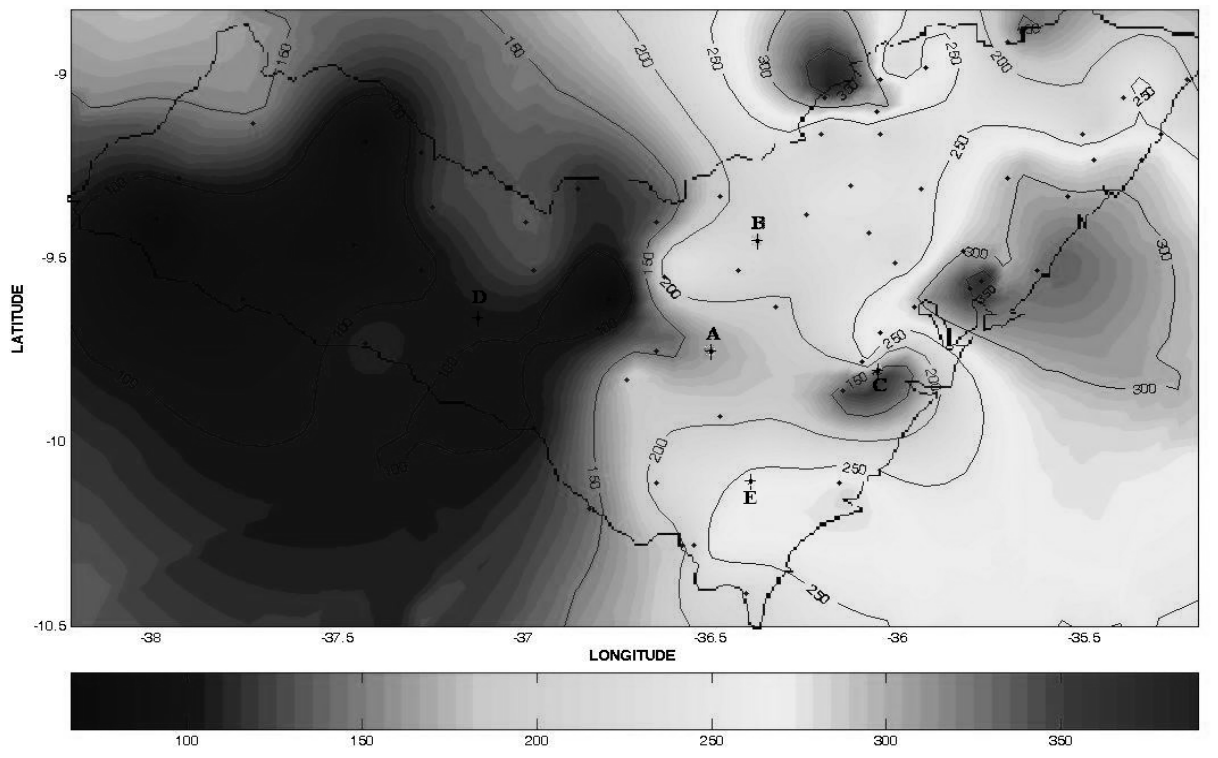

Figura 5 - Interpolação espacial da precipitação obtida por krigagem ordinária para o mês de maio com 63 estações.

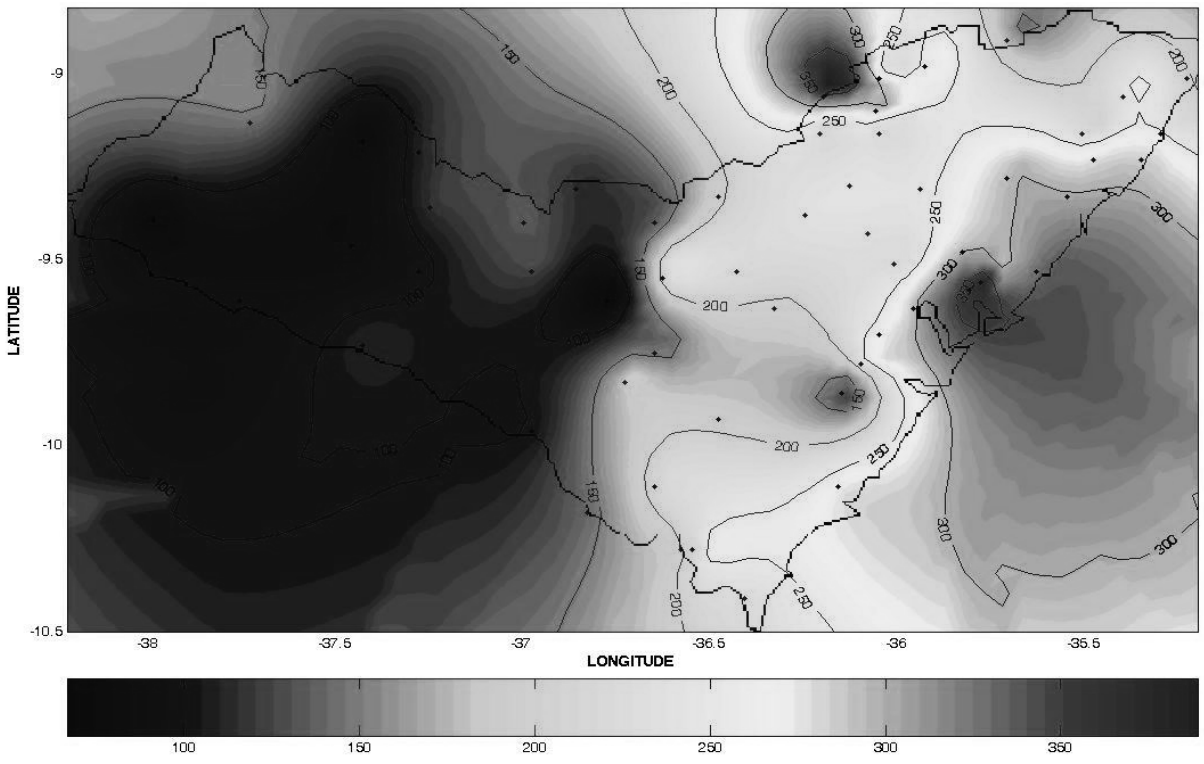

Figura 6 - Interpolação espacial da precipitação obtida por krigagem ordinária para o mês de maio com 58 estações. 
Comrie e Broyles (2002) comentaram que, em geral, a variabilidade da precipitação no verão e inverno está ligada a trocas de calor nas direções zonais e meridionais e aos movimentos no sentido leste-oeste do anticiclone sobre a região. Os autores observaram também que os processos convectivos localizados conduzem as anomalias no verão, sendo especialmente mais complexas que no inverno, quando há presença de sistemas meteorológicos de grande escala.

Devido a menor variabilidade pluviométrica observada no mês de novembro, a estimativa da precipitação para as estações A, B, C, D e E apresentou resultados mais condizentes com a série de dados medidos nas estações (Tabela 3). O mês de novembro apresentou desvio médio de $1,4 \mathrm{~mm}$ e erro quadrático médio de 2,1 mm. A maior diferença entre o valor observado e o estimado foi verificada para a estação E (Figura 8).

\section{CONCLUSÕES}

A utilização da geoestatística com a análise variográfica mostrou que as características estruturais estudadas da precipitação estão correlacionadas e apresentam forte dependência espacial. Os modelos esférico e exponencial foram

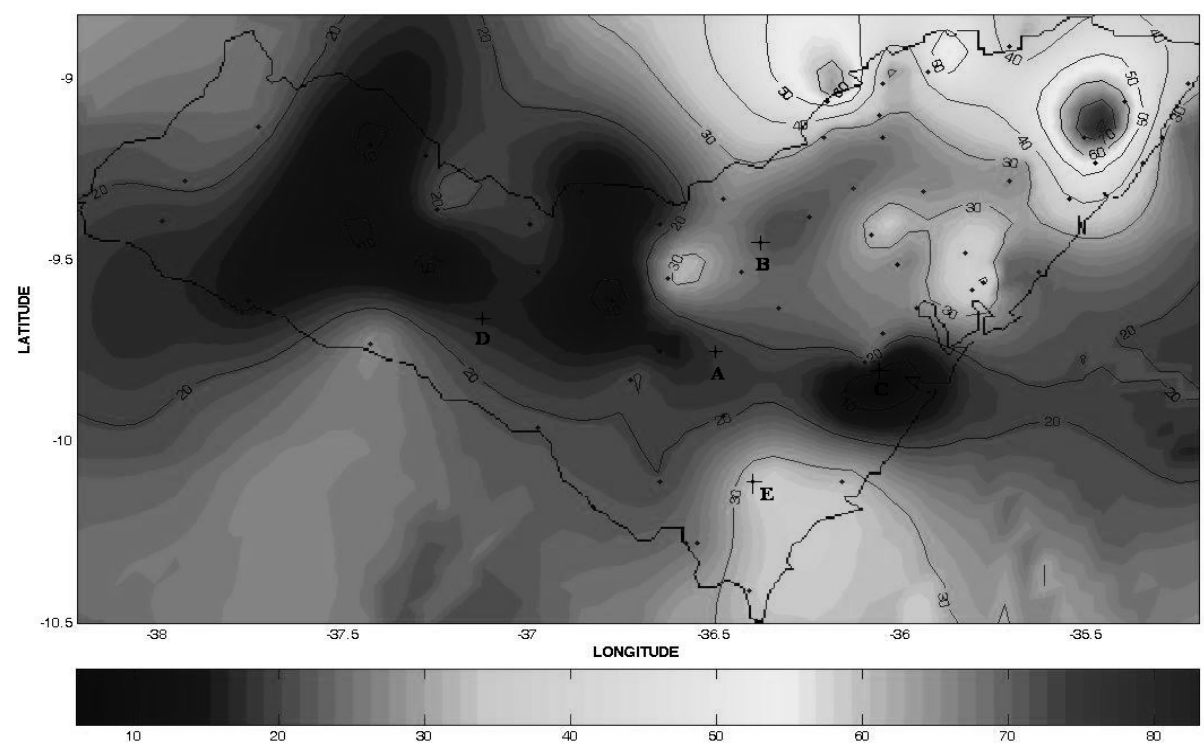

Figura 7 - Interpolação espacial da precipitação obtida por krigagem ordinária para o mês de novembro com 63 estações.

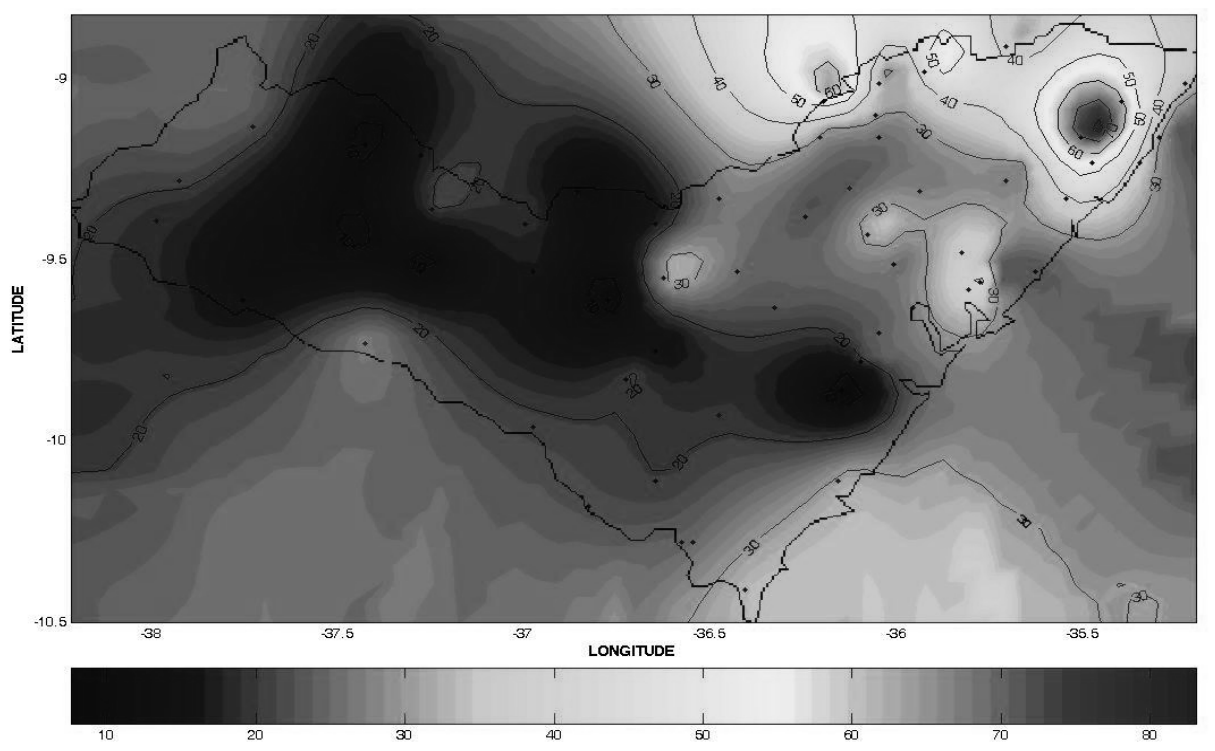

Figura 8 - Interpolação espacial da precipitação obtida por krigagem ordinária para o mês de novembro com 58 estações. 
os que apresentaram um melhor ajuste aos dados de precipitação para maio e novembro, respectivamente. Neste estudo foi possível determinar a região de dependência espacial, definindo assim o limite máximo onde os dados estão correlacionados.

A distribuição da precipitação apresentou grande variabilidade espacial, sendo a região Litorânea e a Zona da Mata, as que apresentaram os maiores índices pluviométricos do Estado. Ao entrar no continente, observou-se uma redução significativa dos totais de pluviométricos.

A estimativa da precipitação obtida por krigagem ordinária apresentou resultados satisfatórios, estando condizentes com as características locais de distribuição das chuvas, tanto na distribuição espacial, quanto sazonal da precipitação. A precipitação estimada para as estações A, B, C, $\mathrm{D}$ e $\mathrm{E}$ apresentaram resultados satisfatórios para ambos os meses em estudo, sendo o mês de maio o que apresentou maior DM e EQM. Os resultados obtidos neste estudo ratificam a eficácia da metodologia proposta para se estimar a precipitação para as regiões que não apresentam estações de medições, ou que apresentam falhas em seu banco de dados.

\section{REFERÊNCIAS BIBLIOGRÁFICAS}

ALSAMAMRA, H.; RUIZ-ARIAS, J. A.; POZO-VÁZQUEZ, D.; TOVAR-PESCADOR, J. A comparative study of ordinary and residual kriging techniques for mapping global solar radiation over southern Spain. Agricultural and Forest Meteorology, v. 149, n. 8, 3, p. 1343-1357, 2009.

BARGAOUI, Z. K.; CHEBBI, A. Comparison of two kriging interpolation methods applied to spatiotemporal rainfall. Journal of Hydrology, v.365, n. 1-2,p. 56-73, 2009.

CAMBARDELLA, C.A., MOORMAN, T.B., NOVAK, J.M., PARKIN,T.B., KARLEN, D. L., TURCO, R.F., KONOPKA, A.E. Field-scale variability of soil properties in Central Iowa soils. Soil Science Society of America Journal. v. 58, p. 1501-11, 1994.

CARVALHO, J. R. P.; VIEIRA, S. R.; GREGO, C. R. Comparação de métodos para ajuste de modelos de semivariograma da precipitação pluvial anua. Revista Brasileira de Engenharia Agrícola e Ambiental, v.13, n.4, páginas 443-448, 2009.

COMRIE, A. C. e BROYLES, B. Variability and spatial modeling of fine-scale precipitation data for the Sonoran Desert of southwest Arizona. University of Arizona, Tucson, U.S.A. Journal of Arid Environments, v. 50, p. 573-592, 2002.

COULIBALY; P., EVORA; N. D. Comparison of neural network methods for infilling missing daily weather records. Journal of Hydrology, v. 341, n. 1-2, 20, p. 27-41, 2007.
HABERLANDT, U. Geostatistical interpolation of hourly precipitation from rain gauges and radar for a large-scale extreme rainfall event. Journal of Hydrology, v. 332, n. 1-2, p. 144-157, 2007.

KUHN, G.; KHAN, S.; GANGULY, A. R.; BRANSTETTER, M. L. Geospatial-temporal dependence among weekly precipitation extremes with applications to observations and climate model simulations in South America. Advances in Water Resources, v. 30, n. 12, p. 2401-2423. 2007.

LANDIM, P. M. B. Sobre Geoestatística e mapas. Terrae Didática. 2(1): pages 19-33, 2006.

LEE, W. K.; BIGING, G. S.; SON, Y.; BYUN, W. H.; LEE, K. H.; SON, Y.M.; SEO, J. H. Geostatistical analysis of regional differences in stem taper form of Pinus densiflora in central Korea. Journal of Ecological Research. Publisher Springer Japan. ISSN 0912-3814, v. 21, n. 4, , p. 144017032006.

LLUYD, C. D. Assessing the effect of integrating elevation data into the estimation of monthly precipitation in Great Britain. Journal of Hydrology. v. 308, n. 1-4, p. 128-150. 2005.

MELLO, C. R.; LIMA, J. M.; SILVA, A. M.; MELLO, J. M.; OLIVEIRA, M. S. Krigragem e Inverso do Quadrado da Distância para a Interpolação dos Parâmetros da Equação de Chuva Intensas. Revista Brasileira de Ciência do Solo, v. 27 p. 925-933, 2003.

MOLION, L. C. B.; BERNADO, S. O. Uma revisão dinâmica das chuvas no Nordeste Brasileiro. Revista Brasileira de Meteorologia, v. 17, n.1, p. 1-10. 2002.

SHAO, X.; STEIN, M.; CHING, J. Statistical comparisons of methods for interpolating the output of a numerical air quality model. Journal of Statistical Planning and Inference, v. 137, n.7-1 p. 2277-2293. 2007.

SIROIS, A. The effects of missing data on the calculation of precipitation-weighted-mean concentrations in wet deposition. Atmospheric Environment. Part A. General Topics, v. 24, n. 9, p. 2277-2288. 1990.

TEEGAVARAPU; R. S. V. Use of universal function approximation in variance-dependent surface interpolation method: An application in hydrology. Journal of Hydrology, v. 332, n.1-2,p. 16-29. 2007.

TEEGAVARAPU, R. S. V. e CHANDRAMOULI V. Improved weighting methods, deterministic and stochastic data-driven models for estimation of missing precipitation records. Journal of Hydrology, v. 312, n. 191-206. 2005.

ZAMBOTI, J. L. Mapas pluviométricos médios mensais no verão para o Estado do Paraná, utilizando métodos geoestatísticos. Tese (Doutorado em Agronomia/Energia na Agricultura) - Faculdade de Ciências Agronômicas, Universidade Estadual Paulista. Botucatu, página 134. 2001. 